\title{
FACTORES SOCIODEMOGRÁFICOS Y GESTACIONALES ASOCIADOS A LA CONCENTRACIÓN DE HEMOGLOBINA EN EMBARAZADAS DE LA RED HOSPITALARIA PÚBLICA DE MEDELLÍN
}

\author{
SOCIODEMOGRAPHIC AND PREGNANCY ASPECTS \\ ASSOCIATED WITH HEMOGLOBIN CONCENTRATION IN \\ WOMEN FROM PUBLIC HOSPITALS IN MEDELLÍN
}

\author{
Luz Stella Escudero V., Beatriz Elena Parra S., Sandra Lucía Restrepo M. \\ Escuela de Nutrición y Dietética \\ Universidad de Antioquia, Colombia.
}

\begin{abstract}
Objective: To explore associations of sociodemographic and gestational factors with blood hemoglobin concentration. Methodology: Randomized sample of 336 pregnant women medical records from the Public Hospital Network in Medellín. Hemoglobin concentration, sociodemographic, and pregnancy data analyzed by Student T test, ANOVA and Pearsons correlation. Results: Average age of pregnant women was 24 $\pm 6 ; 3.3 \% ; 67.6 \%$ of them were high school graduates; $62.2 \%$ had permanent partners. Number of attendance to prenatal appointments was $6 \pm 1$. Pregestacional weight was $54.4 \pm 8.2 \mathrm{~kg}$; average hemoglobin concentration on the first trimester was $13.0 \pm 1.0 \mathrm{~g} / \mathrm{dl}, 11.9 \pm 1.1 \mathrm{~g} / \mathrm{dl}$ on the second, and 12.2 $\pm 1.1 \mathrm{~g} / \mathrm{dl}$ on the third. There was a significant difference in hemoglobin concentration based on pregestacional body mass index (BMI) $(p=0,035)$, a significant correlation between first trimester hemoglobin concentration and pregestacional BMI $(r=0.178, p=0.028)$, and also with third trimester hemoglobin concentration ( $r=0.356$ and $p=0.000$ ). Conclusion: Pregestacional BMI was associated with hemoglobin concentration at the beginning and at the end of pregnancy.
\end{abstract}

Key words: Pregnancy, hemoglobin, anemia, social conditions, prenatal care.

Este trabajo fue recibido el 20 de Abril de 2011 y aceptado para ser publicado el 1 de Agosto de 2011.

\section{INTRODUCCIÓN}

El embarazo es una de las etapas del proceso vital humano con mayores demandas de hierro (Fe) y cambios hemodinámicos importantes, que hacen a la mujer más susceptible a variaciones en la concentración de hemoglobina $(\mathrm{Hb})$, hasta alcanzar valores no fisiológicos que comprometen su salud, asociándose con mal resultado materno-fetal(1-4). La deficiencia de Fe que afecta la producción de $\mathrm{Hb}$ y desencadena anemia $(\mathrm{Hb}<11 \mathrm{~g} /$ $\mathrm{dL}$ ), incide negativamente en el sistema inmune y dificulta el transporte de oxígeno a los tejidos, con graves consecuencias para el feto, aumento en el riesgo de prematuridad, bajo peso al nacer y morbimortalidad materno-infantil $(5,6)$. Por otra parte, algunas patologías de importante prevalencia en la gestación, como el síndrome hipertensivo asociado al embarazo (SHAE) y la diabetes mellitus, cursan con aumento en la concentración de $\mathrm{Hb}$ y son consideradas una seria amenaza para el binomio madre-hijo (7).

Se han estudiado los factores alimentarios y nutricionales que afectan la concentración de $\mathrm{Hb}$ gestacional, relacionados con los altos requerimientos de esta etapa, deficiente ingesta de micronutrientes, bajo contenido y/o biodisponibilidad de $\mathrm{Fe}$ y ácido fólico, además la no utilización oportuna y adecuada de un suplemento con $\mathrm{Fe}$, que predisponen a la mujer a su deficiencia y al desarrollo de anemia (8).

Algunos estudios han evidenciado asociación de factores maternos no nutricionales, como sociodemográficos y gestacionales, con la concentración de $\mathrm{Hb}$.

Investigación realizada en: La Red Hospitalaria Pública de Medellín, Colombia. 
En cuanto a los sociodemográficos se citan: la edad materna, escolaridad, etnia, estado civil y régimen de afiliación a seguridad social (9). En los pregestacionales se han considerado: el número de embarazos previos, período intergenésico y peso pregestacional, y en los gestacionales: ingreso oportuno a controles prenatales (CPN), número de éstos, seguimiento a la ingesta de suplementos y a la $\mathrm{Hb}$ (9-11). Los factores anteriormente mencionados, comprometen las reservas maternas de $\mathrm{Fe}$, otros micronutrientes importantes en la eritropoyesis y la concentración de $\mathrm{Hb}$ (2-5); algunos de estos aspectos pueden asociarse más fuertemente con la $\mathrm{Hb}$ gestacional que otros(8), y en la medida que se reconozcan, podrán elaborarse protocolos de atención y seguimiento a embarazadas con alto riesgo, previniendo el descenso no fisiológico en los valores de $\mathrm{Hb}$ y por ende la anemia, como también, valores anormalmente altos asociados con patologías.

Es importante anotar, que si bien la alta concentración de $\mathrm{Hb}$ preocupa por su relación con algunas patologías en la gestación, los bajos niveles son el problema más prevalente durante el embarazo; ante dicha situación, entes gubernamentales y salubristas del mundo, se preocupan por las graves consecuencias que acarrean la baja concentración de $\mathrm{Hb}$ y el desarrollo de anemia materna para la salud gestacional y posnatal; esto los ha llevado a fortalecer acciones estratégicas para prevenir la deficiencia de Fe y la anemia $(\mathrm{Hb}<11 \mathrm{~g} / \mathrm{dL})$, por ser éstos dos problemas de gran costo social y económico que afectan el bienestar materno-infantil y prevenibles con opciones de bajo costo y alta efectividad (9).

En Colombia, la anemia y la ferropenia en gestantes son problemas de salud pública, situación que se agrava por la relación del pobre estado de hierro con la mortalidad materna, la cual alcanzó una razón de 42 muertes por cada 100.000 nacidos vivos en Medellín para 2010. Según la ENSIN 2005, 32,8\% del total de embarazadas presentó anemia y $69.9 \%$ tenía algún grado de ferropenia. Lo anterior refleja un limitado seguimiento a las embarazadas y la falta de decisiones oportunas que contribuyan a la detección temprana de alteraciones en la gestación, tal como lo establece la resolución 412 de 2000 del Ministerio de Salud, que indica que las embarazadas deben tener al menos, dos exámenes de $\mathrm{Hb}$ durante este período. Sin embargo, se encuentra que un gran número de ellas sólo tiene uno, lo que demuestra que el seguimiento a este indicador, es de poca importancia para el personal médico, quien reacciona con medidas curativas únicamente cuando la concentración cae en el rango de anemia, en lugar de prevenir la deficiencia, basándose en los factores sociodemográficos de riesgo. Chile y Cuba han superado este problema pero en Colombia, es aun prevalente la situación por lo que se justifica el presente trabajo en pro de la búsqueda de factores que se asocien a la concentración de hemoglobina y permitan acciones para prevenir su descenso patológico durante el embarazo y la promoción de la salud materna y del niño.

Varias investigaciones sobre factores que inciden en la $\mathrm{Hb}$ gestacional, están enfocadas al estudio de las causas de anemia y sus efectos en la salud maternofetal y neonatal; sin embargo, pocas buscan explicar la concentración de la $\mathrm{Hb}$, en relación con los factores sociodemográficos y gestacionales que afectan los valores séricos de dicha proteína.

Por lo anterior, el objetivo de este estudio fue analizar aspectos sociodemograficos y gestacionales asociados a la concentración de $\mathrm{Hb}$ materna, para sensibilizar y promover en los sectores de salud y desarrollo comunitario, acciones que mejoren ó mantengan el estado nutricional materno y la prevención de la malnutrición desde las áreas social, cultural y clínica en instituciones que atienden este grupo poblacional, altamente vulnerable.

\section{SUJETOS Y MÉTODOS}

Se realizó un estudio descriptivo de corte transversal en una población constituida por 1145 historias clínicas de gestantes, con controles prenatales (CPN) e hijos nacidos vivos, que consultaron durante 2007 , en una misma Unidad Hospitalaria (UH) de las siete evaluadas, pertenecientes a la Red Hospitalaria Pública de Medellín (Colombia), que atiende una población en situación vulnerable, en su mayoría de niveles 1,2 y 3 del SISBEN (Sistema de Identificación de Potenciales Beneficiarios de Programas Sociales).

Este estudio se basó en los datos obtenidos del proyecto marco "Estado del hierro, índice de masa corporal materno y su relación con el peso al nacer del neonato", en el cual, para el cálculo del tamaño de muestra, se tuvieron en cuenta, los siguientes parámetros: la $\mathrm{Hb}$ materna como variable de interés, una desviación estándar de 1,0 g/dL, según datos obtenidos en el proyecto de investigación: "Estado nutricional de un grupo de mujeres gestantes y de sus recién nacidos que reciben complementación alimentaria, suplemento de micronutrientes y educación nutricional. Subregión Bajo Cauca, 2006" (12), con una confianza de $95 \%$ y un error de muestreo de $0,1 \mathrm{~g} / \mathrm{dL}$, se obtuvo un tamaño de muestra de 291 historias clínicas prenatales; a esta muestra se le aplicó $15 \%$ de sobremuestreo, para recuperar la pérdida de información debida a historias clínicas incompletas, mal diligenciadas o ilegibles y obtener finalmente un total de 336 historias. Para este cálculo se utilizó el 
programa Epidat versión 3,1.

La selección de la muestra se realizó mediante muestreo aleatorio estratificado por UH, tomando como marco muestral el listado de las historias clínicas de mujeres que hubieran tenido CPN y parto en la misma UH, suministrado por el departamento de estadística de la Red Hospitalaria Pública de Medellín.

Los criterios de inclusión fueron: gestantes hasta 40 años, que hubieran tenido un hijo vivo, a término, en la Red Hospitalaria Pública de Medellín durante 2007, sanas, con ingreso al programa de CPN en el primero o segundo trimestre de gestación, con asistencia como mínimo a tres controles, con gestación de un sólo feto, sin uso de sustancias psico-activas, cigarrillo, licor u otras y con historias clínicas completas, sin errores en los registros como tachones, enmendaduras, ilegibilidad e inconsistencia de los datos.

Se utilizó un formulario precodificado para recolectar las variables de interés, a partir de las historias clínicas prenatal y perinatal, propuestas por el Centro Latinoamericano de Perinatología y Desarrollo Humano (CLAP), avaladas por la Dirección Seccional de Salud de Antioquia (DSSA) y utilizadas por la Red Hospitalaria Pública de Medellín. Dichas historias fueron diligenciadas por el personal de salud de cada UH, consignando la información sociodemográfica y de salud de la madre cuando ingresó al programa y la evolución del embarazo, parto y postparto inmediato. Los datos de $\mathrm{Hb}$ también se obtuvieron de la historia prenatal y el método empleado en su determinación fue el de la cianometahemoglobina modificado, en equipos de lectura automática marca Celltak-auto-Nihon Kohden modelo Mek 8118 y Advia 60; pese a que la ciudad de Medellín, se encuentra ubicada a 1500 m.s.n.m, no se aplicó el factor de corrección en la concentración de $\mathrm{Hb}$ de las gestantes por la altura, debido a que el interés del estudio no es clasificar las embarazadas como anémicas o no anémicas con relación a un punto de corte definido sobre el nivel del mar (11 g/ dL) sino, asociar las diferentes concentraciones de $\mathrm{Hb}$ en la embarazada con factores sociodemograficos y gestacionales. Con los datos de estatura y peso pregestacional materno, obtenidos de la historia, se calculó el Índice de Masa Corporal (IMC) y los resultados se clasificaron por los puntos de corte sugeridos por Atalah y colaboradores según las semanas de gestación: IMC $<20 \mathrm{~kg} / \mathrm{m}^{2}$ (bajo), IMC entre 20 y $25 \mathrm{~kg} / \mathrm{m}^{2}$ (adecuado), IMC $>25 \mathrm{~kg} / \mathrm{m}^{2}$ (exceso de peso) (10). Se utilizó el gestograma para calcular las semanas de gestación que tenía la madre al momento de determinar la $\mathrm{Hb}$ y el peso materno. Para valorar la ingesta del suplemento de hierro y por tratarse de fuentes secundarias, se hizo un análisis detallado de cada control prenatal registrado en la historia clínica y se establecieron las siguientes categorías para identificar el seguimiento que el profesional de la salud hace al consumo del suplemento: adecuado cuando en el $100 \%$ de controles prenatales a los que asistió la gestante, se hacía alguna referencia al suplemento, aceptable cuando esta nota estaba en 80 a 99\% de los CPN e inadecuado al encontrar que en menos del $80 \%$ de los CPN, se anotaba alguna observación al suplemento.

Con el fin de garantizar la calidad del dato, se realizó una prueba piloto en una UH de la Red Hospitalaria Pública de Medellín, donde se aplicó el instrumento de recolección de la información a 48 historias prenatales y perinatales para evaluar los procedimientos en la toma de datos y considerar la calidad, claridad y pertinencia de los datos recolectados, manejo del tiempo por parte de los investigadores responsables del trabajo de campo, quienes fueron previamente estandarizados en la recolección de la información.

Se realizaron controles para evitar posibles sesgos desde el observador, mediante la estandarización para recolectar la información y visitas de supervisión; desde los instrumentos de medición, con formularios debidamente precodificados y sometidos a la prueba piloto para ajustes definitivos y finalmente, desde los observados, por el conocimiento previo de las historias clínicas.

La base de datos y el análisis estadístico se realizó en el software SPSS versión 17. El análisis descriptivo incluyó medidas de tendencia central y dispersión como media, mediana y desviación estándar para las variables numéricas y porcentaje para las cualitativas.

Se asumió como variable dependiente la concentración de $\mathrm{Hb}$ gestacional; en los casos en que la madre tuvo varios datos, se tomó aquel más cercano al final de cada trimestre de gestación, como el valor a tener en cuenta en los análisis ya que se asume que refleja la condición de dicha proteína al final de cada trimestre.

Con el test de Kolmogorov-Smirnov, se determinó la normalidad en la distribución de la Hb en cada trimestre gestacional. Se hizo un análisis bivariado buscando una asociación de la concentración de $\mathrm{Hb}$ con variables explicativas como edad materna, nivel de escolaridad, estado civil, número de gestas previas, período intergenésico, número de CPN e IMC pregestacional. Se aplicaron las pruebas $\mathrm{T}$ de Student para grupos independientes al comparar las concentraciones de Hb según edad, estado civil y nivel educativo, ANOVA con pos-test Tukey para comparación de $\mathrm{Hb}$ con variables como IMC pregestacional y período intergenésico. Se utilizó la correlación de Pearson para estimar la asociación de variables como número de gestas previas, peso pregestacional, total de CPN e IMC pregestacional, con la concentración de $\mathrm{Hb}$ de primer y tercer trimestre por considerar que son estos 
valores, quienes reciben finalmente el peso de las variables pregestacionales y gestacionales. Para el control de las variables de confusión como nivel educativo, estado civil y edad, se utilizó un análisis de regresión múltiple. Se definió como significancia una $\mathrm{p}<0.05$.

Aspectos éticos: por ser un estudio con fuente de datos secundaria, se clasificó sin riesgo, de acuerdo con la resolución 8430 del Ministerio de Salud (14). Este proyecto contó con el aval del comité de ética de la Red Hospitalaria Pública de Medellín.

\section{RESULTADOS}

La edad promedio de las gestantes fue $24 \pm 6$ años, $14,9 \%$ correspondió a adolescentes y $85,1 \%$ a adultas; $27,1 \%$ tenía estudios completos o incompletos de primaria, 67,6\% con algún grado de secundaria y 3,3\% con educación superior; $62,2 \%$ tenía compañero estable, siendo más en unión libre que casadas; asistieron en promedio a $6 \pm 1 \mathrm{CPN}$, con un rango de tres a doce, $50,6 \%$ asistió a seis o siete CPN, mientras que $15,2 \%$, tuvo ocho o más controles.

Respecto al peso pregestacional, destaca que debido al ingreso tardío a los CPN, el 19\% de las mujeres no tenían este dato y del $81 \%$ de historias prenatales que contaron con el registro de peso pregestacional $(54,4 \pm 8,2$ $\mathrm{kg}$ ), de este porcentaje, $55,4 \%$ registró entre 46 y $60 \mathrm{~kg}$; $10,4 \%$ de las madres empezó su embarazo con un peso igual o inferior a los $45 \mathrm{~kg}$.
La media de $\mathrm{Hb}$ para el grupo de embarazadas fue de $13,0 \pm 1,0 \mathrm{~g} / \mathrm{dL}, 11,9 \pm 1,1 \mathrm{~g} / \mathrm{dL}$ y $12,2 \pm 1,1 \mathrm{~g} / \mathrm{dL}$ en el primero, segundo y tercer trimestre de embarazo respectivamente. Los valores mínimo y máximo de $\mathrm{Hb}$ en el primer trimestre fueron $10,4 \mathrm{~g} / \mathrm{dL}$ y $15,7 \mathrm{~g} / \mathrm{dL}$, en segundo $8,3 \mathrm{~g} / \mathrm{dL}$ y $15,7 \mathrm{~g} / \mathrm{dL}$ y en el tercero de $9,1 \mathrm{~g} /$ dL y $15,9 \mathrm{~g} / \mathrm{dL}$.

La media de $\mathrm{Hb}$ no presentó diferencias significativas por trimestre de gestación de acuerdo con la edad, el nivel educativo y el estado civil de las embarazadas (tabla 1). Las madres que empezaron su embarazo enflaquecidas (IMC $<20$ ) tuvieron valores de $\mathrm{Hb}$ más bajos en el primer trimestre $(p=0,035)$ que sus contrapartes de peso normal o en exceso; en el tercer trimestre, a pesar de no existir diferencias significativas $(\mathrm{p}=0,052)$, estas mujeres presentaron niveles más bajos de $\mathrm{Hb}$, en relación a los otros grupos. Las gestantes con IMC pregestacional normal, terminaron la gestación con mejor valor de $\mathrm{Hb}$, comparadas con las enflaquecidas y con las de exceso de peso, aunque sin diferencias significativas (tabla 2). Por paridad y período intergenésico tampoco se hallaron diferencias significativas en la $\mathrm{Hb}$, en ningún trimestre de la gestación, sin embargo, aquellas mujeres con intervalo intergenésico $\leq 24$ meses, iniciaron el embarazo con valores de Hb más bajos que aquellas en las categorías de 25 a 60 meses ó mayores de 61 meses (tabla 2).

La media de Hb no presentó variaciones significativas según el número de $\mathrm{CPN}$ a los que asistió la madre,

\section{TABLA 1}

Concentración de hemoglobina por trimestre de gestación de acuerdo con variables sociodemográficas maternas.

\begin{tabular}{|c|c|c|c|c|c|c|c|c|c|}
\hline \multirow[b]{2}{*}{ VARIABLES } & \multicolumn{3}{|c|}{ Trimestre 1} & \multicolumn{3}{|c|}{ Trimestre 2} & \multicolumn{3}{|c|}{ Trimestre 3} \\
\hline & n & $\mathrm{Hb}^{*}$ & $\begin{array}{c}\mathbf{p}^{* *} \\
(\text { IC } 95 \%)\end{array}$ & $\mathbf{n}$ & $\mathrm{Hb}$ & $\begin{array}{c}\mathbf{p} \\
(\text { IC 95\%) }\end{array}$ & n & $\mathrm{Hb}$ & $\begin{array}{c}p \\
(\text { IC 95\%) }\end{array}$ \\
\hline \multicolumn{10}{|l|}{ Edad $†$} \\
\hline 14-18 Años & 23 & $12,9 \pm 0,8$ & 0,543 & 29 & $11,8 \pm 1,2$ & 0,604 & 25 & $12,2 \pm 1,1$ & 0,974 \\
\hline 19 - 40 Años & 162 & $13,0 \pm 1,0$ & $(-0,57 ; 0,30)$ & 140 & $12,0 \pm 1,1$ & $(-0,58 ; 0,34)$ & 181 & $12,2 \pm 1,1$ & $(-0,47 ; 0,48)$ \\
\hline \multicolumn{10}{|l|}{ Nivel educativo $\dagger$} \\
\hline Primaria ó menos & 58 & $13 \pm 0,9$ & 0,705 & 43 & $12,0 \pm 1,0$ & 0,619 & 54 & $12,1 \pm 1,1$ & 0,779 \\
\hline Secundaria ó más & 126 & $13,1 \pm 1,0$ & $(-0,37 ; 0,25)$ & 126 & $11,9 \pm 1,2$ & $(-0,30 ; 0,50)$ & 152 & $12,2 \pm 1,2$ & $(-0,41 ; 0,30)$ \\
\hline \multicolumn{10}{|l|}{ Estado civil $†$} \\
\hline Soltera & 74 & $13,0 \pm 0,9$ & 0,709 & 56 & $12,1 \pm 1,3$ & 0,213 & 75 & $12,2 \pm 1,2$ & 0,740 \\
\hline Casada/unión libre & 110 & $13,0 \pm 1,0$ & $(-0,35 ; 0,24)$ & 113 & $11,9 \pm 1,0$ & $(-0,13 ; 0,60)$ & 130 & $12,2 \pm 1,1$ & $(-0,27 ; 0,38)$ \\
\hline
\end{tabular}


pero se observaron valores más elevados, en el segundo y tercer trimestre, en quienes asistieron a ocho ó más controles (tabla 2).

En 14\% de las historias prenatales se registró en cada CPN, alguna información referente al suplemento de $\mathrm{Fe}$, mientras que en $67 \%$ de ellas se hizo alusión al suplemento en un $80 \%$ de los CPN. Los promedios de $\mathrm{Hb}$ por trimestre no presentaron diferencias significativas según la clasificación del seguimiento a la ingesta del suplemento de $\mathrm{Fe}(\mathrm{p}>0,05)$ (tabla 2).

Se halló una correlación positiva entre la $\mathrm{Hb}$ de primer trimestre tanto con el peso corporal pregestacional como con el IMC pregestacional, igualmente se encontró entre la concentración de $\mathrm{Hb}$ inicial y la del tercer trimestre. No hubo correlación significativa entre la edad de las gestantes, el número de gestas previas y de $\mathrm{CPN}$, con las concentraciones de $\mathrm{Hb}$ del primer ni del tercer trimestre de gestación (tabla 3).

Se utilizó la regresión múltiple para controlar las correlaciones anteriores según nivel educativo, estado civil y edad de la madre, encontrando después de construir un modelo con la $\mathrm{Hb}$ de primer trimestre como variable dependiente y las variables peso pregestacional e IMC pregestacional acompañadas del nivel educativo, estado civil y edad de la madre como variables independientes, que la asociación entre la $\mathrm{Hb}$ inicial con el peso pregestacional $(\mathrm{p}=0,027)$ y el IMC pregestacional $(\mathrm{p}=0,032)$, seguían siendo similares después de ajustar por estas variables. Situación similar se halló cuando se construyó un modelo para $\mathrm{Hb}$ de tercer trimestre como variable dependiente, asociada con la $\mathrm{Hb}$ de primer trimestre y las variables nivel educativo, estado civil y edad de la madre como variables independientes, reflejando que la presencia de estas variables sociodemográficas no

\section{TABLA 2}

\section{Concentración de hemoglobina por trimestre de gestación de acuerdo con variables gestacionales maternas.}

\begin{tabular}{|c|c|c|c|c|c|c|c|c|c|}
\hline \multirow[t]{2}{*}{ VARIABLES } & \multicolumn{3}{|c|}{ Trimestre 1} & \multicolumn{3}{|c|}{ Trimestre 2} & \multicolumn{3}{|c|}{ Trimestre 3} \\
\hline & $\mathbf{n}$ & $\mathbf{H b}^{*}$ & $\mathbf{p}^{* *}$ & $\mathbf{n}$ & $\mathbf{H b}$ & $\mathbf{p}$ & $\mathbf{n}$ & $\mathbf{H b}$ & $\mathbf{p}$ \\
\hline \multicolumn{10}{|l|}{$\begin{array}{l}\text { IMC } \\
\text { pregestacional } \neq\end{array}$} \\
\hline Enflaquecida & 32 & $12,7 \pm 1,0 \mathrm{a}$ & 0,035 & 33 & $12,0 \pm 1,1$ & 0,942 & 40 & $11,9 \pm 1,1$ & 0,052 \\
\hline Normal & 85 & $13,1 \pm 1,0 \mathrm{~b}$ & & 82 & $12,0 \pm 1,3$ & & 96 & $12,4 \pm 1,2$ & \\
\hline Exceso de peso & 35 & $13,2 \pm 0,8 \mathrm{~b}, \mathrm{c}$ & & 15 & $12,1 \pm 0,8$ & & 31 & $12,1 \pm 1,1$ & \\
\hline \multicolumn{10}{|l|}{$\begin{array}{l}\text { Período } \\
\text { intergenesico } \uparrow\end{array}$} \\
\hline$\leq 24$ meses & 34 & $12,9 \pm 1,0$ & 0,660 & 57 & $12,1 \pm 1,0$ & 0,951 & 61 & $12,2 \pm 1,2$ & 0,603 \\
\hline 25 - 60 meses & 32 & $13,1 \pm 1,0$ & & 28 & $12,0 \pm 1,4$ & & 29 & $12,2 \pm 1,2$ & \\
\hline$\geq 61$ meses & 33 & $13,1 \pm 1,2$ & & 21 & $12,1 \pm 1,1$ & & 37 & $12,0 \pm 1,1$ & \\
\hline \multicolumn{10}{|l|}{$\begin{array}{l}\text { Total controles } \\
\text { prenatales } \ddagger\end{array}$} \\
\hline 3 a 5 & 22 & $13,1 \pm 1,2$ & 0,926 & 91 & $12,0 \pm 1,1$ & 0,672 & 60 & $12,2 \pm 1,2$ & 0,770 \\
\hline 6 a 7 & 118 & $13,0 \pm 1,0$ & & 66 & $11,9 \pm 1,1$ & & 104 & $12,1 \pm 1,2$ & \\
\hline 8 y más & 45 & $13,1 \pm 0,8$ & & 12 & $12,2 \pm 1,7$ & & 42 & $12,3 \pm 0,9$ & \\
\hline \multicolumn{10}{|l|}{$\begin{array}{l}\text { Seguimiento de } \\
\text { hierro } \neq\end{array}$} \\
\hline Adecuado (100\%) & 20 & $13,1 \pm 0,9$ & 0,570 & 28 & $12,0 \pm 1,1$ & 0,452 & 30 & $12,2 \pm 1,1$ & 0,223 \\
\hline Aceptable $(80-99,9 \%)$ & 40 & $12,9 \pm 1,0$ & & 30 & $11,7 \pm 1,3$ & & 45 & $11,9 \pm 1,1$ & \\
\hline Inadecuado $(<80 \%)$ & 125 & $13,1 \pm 1,0$ & & 111 & $12,0 \pm 1,1$ & & 131 & $12,3 \pm 1,1$ & \\
\hline $\begin{array}{l}\text { † Análisis de varianza. } \\
\text { * Valor promedio de Hemo } \\
\text { ** Valor p (intervalo de con } \\
\text { Letras distintas indican difer }\end{array}$ & $\begin{array}{l}a \pm c \\
\text { de } 1\end{array}$ & $\begin{array}{l}\text { iación están } \\
\text { erencia de } 1 \\
\text { ativas. }\end{array}$ & dias al & & & & & & \\
\hline
\end{tabular}


modificaron el comportamiento de la asociación entre la $\mathrm{Hb}$ del tercer trimestre y la del primero $(\mathrm{p}=0,000)$.

\section{DISCUSIÓN}

El objetivo del presente estudio fue analizar factores socio-demográficos y gestacionales determinantes de la concentración de hemoglobina materna en las gestantes de la Red Hospitalaria Pública de Medellín. Al respecto, se encontró que el estado nutricional previo al embarazo fue el aspecto que más intervino en la concentración de $\mathrm{Hb}$ del primer trimestre y que ésta a su vez correlacionó con la $\mathrm{Hb}$ al final de la gestación.

Características como bajo nivel educativo, monoparentalidad, ingreso tardío a CPN, indican vulnerabilidad social y económica de las gestantes atendidas en la Red Hospitalaria Pública de Medellín, siendo además, hechos relacionados con el estado nutricional y la salud materno-fetal, dado el hecho que pueden aumentar el riesgo de presentar bajos niveles de $\mathrm{Hb}$ con consecuencias de morbimortalidad, bajo peso al nacer y retraso psicomotor (11).

La media de $\mathrm{Hb}$ para este grupo, mostró un comportamiento fisiológico acorde con los cambios hemodinámicos del embarazo, con concentraciones más bajas en el segundo trimestre de gestación, por la expansión del volumen plasmático y la depleción de las reservas férricas e incremento en tercer trimestre, que evidencia una recuperación parcial de la concentración, hacia el final del embarazo (13).

Los cambios en la concentración de $\mathrm{Hb}$ materna pueden estar influenciados por factores fisiológicos, nutricionales (14), sociodemográficos y gestacionales $(6,15)$. En este estudio, no se encontró asociación de la Hb con las características sociodemográficas: edad, nivel educativo y estado civil, de las gestantes en ningún trimestre del embarazo; una posible explicación, es que dichas características en la población atendida por la Red Hospitalaria Pública de Medellín son muy homogéneas.

La edad no fue un factor determinante en la concentración de $\mathrm{Hb}$, probablemente debido a que se prioriza a la adolescente, según normatividad nacional, en un programa de alto riesgo obstétrico que minimiza problemas durante la gestación. Existen trabajos en los cuales tampoco reportan la edad como determinante de la $\mathrm{Hb}(12,15-18)$, y discuten que la atención brindada a la gestante adolescente suele ser priorizada por el riesgo que representa el embarazo en las menores de edad (19). Contrario a este hallazgo, otros autores han demostrado importante relación de la $\mathrm{Hb}$ materna con la edad, al asociar altos requerimientos de Fe con bajas concentraciones de $\mathrm{Hb}$, comunes en la adolescencia $(16,19)$; otra explicación, son los hábitos alimentarios inadecuados y baja adherencia a los suplementos nutricionales debidos al menor nivel educativo y la poca responsabilidad de la adolescente frente a la gestación.

Aun cuando el nivel educativo tampoco se asoció con la $\mathrm{Hb}$ materna, se observó que las gestantes con menos escolaridad, tuvieron valores inferiores respecto a las de mejor condición educativa y coincide esto con otros autores, al sostener que a más baja escolaridad hay menor acceso a los medios de información, al adecuado uso de los suplementos y más deficiencia en la calidad de la alimentación $(15,19,20)$.

El estado civil no fue un factor determinante de la $\mathrm{Hb}$ en este estudio y aunque las investigaciones al respecto consideran esta variable, pocas muestran aso-

\section{TABLA 3}

\section{Correlación entre variables sociodemográficas y gestacionales con la hemoglobina de primer y tercer trimestre.}

\begin{tabular}{lccccccc} 
Variables & \multicolumn{3}{c}{ Hemoglobina $\mathbf{1}$ trimestre } & \multicolumn{3}{c}{ Hemoglobina 3 trimestre } \\
& n & r* & $\mathbf{p}$ & n & r & p \\
Edad & 185 & 0,056 & 0,450 & 206 & $-0,112$ & 0,109 \\
Gestaciones previas & 185 & 0,060 & 0,420 & 206 & $-0,059$ & 0,399 \\
Peso pregestacional & 154 & 0,184 & 0,022 & 170 & $-0,004$ & 0,958 \\
Índice de Masa Corporal Pregestacional & 152 & 0,178 & 0,028 & 167 & 0,039 & 0,612 \\
Hemoglobina 1 trimestre & 185 & & & 112 & 0,356 & 0,000 \\
Total controles prenatales & 185 & 0,005 & 0,947 & 206 & 0,015 & 0,831
\end{tabular}


ciación significativa, salvo por evidenciar el alto índice de mujeres gestantes solteras o convivientes (21); sin embargo, autores como Sotero (22), sostienen que es una variable demográfica importante, dado, que tener una pareja permanente, se manifiesta en la gestante como un estímulo para su autocuidado.

El IMC pregestacional, expresa el estado nutricional previo a la gestación e influye en gran parte en el adecuado desarrollo del embarazo $(4,10)$; las madres que empezaron enflaquecidas tuvieron Hb más baja en el primer y tercer trimestre comparadas con las de peso normal; este hecho coincide con estudios que demuestran la relación entre un buen estado nutricional pregestacional con concentraciones de $\mathrm{Hb}$ adecuadas para afrontar la gestación $(12,15,17)$; de ahí la importancia de garantizar una alimentación óptima en el ciclo reproductivo de la mujer y si es necesario, la utilización de suplementos nutricionales con miras a lograr que la madre alcance el peso y estado nutricional gestacional adecuado antes, durante y después de la gestación $(5,15,17,23)$.

Aunque el estudio no halló diferencias significativas en la $\mathrm{Hb}$ por intervalo intergenésico, se observaron a la par de otras investigaciones $(2,16)$, concentraciones más bajas en embarazadas con períodos intergenésicos inferiores a 24 meses; la ausencia de diferencias estadísticas, pudo deberse al cálculo del tamaño de la muestra.

El sustento científico a estos resultados está dado por el hecho de que las mujeres con períodos intergenésicos cortos, no alcanzan a recuperar el estado nutricional del hierro, afectado por las demandas del embarazo y la lactancia antes de una nueva gestación; siendo más susceptibles a la disminución en la concentración de $\mathrm{Hb}$ y al riesgo de morbimortalidad materno fetal $(8,12)$.

El descenso anormal de la $\mathrm{Hb}$ y el desarrollo de anemia en la gestación, son alteraciones que pueden prevenirse, diagnosticarse y tratarse, con un adecuado CPN $(17,24,25)$. El valor medio de $\mathrm{Hb}$ en las gestantes del estudio, no tuvo variaciones significativas según el número de $\mathrm{CPN}$, sin embargo destacaron mejores niveles en aquellas que asistieron a ocho ó más controles, lo cual puede explicarse como respuesta a la mayor permanencia en el programa. La Sociedad de Ginecología y Obstetricia de Venezuela, entre otras, consideran que la mortalidad perinatal es prevenible al mejorar el número de CPN, la calidad en la atención, el seguimiento oportuno de las recomendaciones y las prescripciones hechas antes, durante y después del parto $(17,26)$. Llama la atención que en los estudios reportados no se especifican los términos de cantidad y/o calidad de los CPN para evaluar dicha variable.

A pesar que las madres del estudio, no presentaron diferencias significativas en los valores de $\mathrm{Hb}$ por trimes- tres, según seguimiento al suplemento de $\mathrm{Fe}$, vale la pena mencionar que el monitoreo a la ingesta y tolerancia del suplemento, es importante para asegurarse que la madre lo tome regularmente, en las dosis adecuadas con el fin de prevenir la deficiencia de Fe y anemia $(16,17)$. Es importante anotar que el seguimiento a la ingesta del suplemento en este estudio, se evaluó de una manera indirecta y por lo tanto no permitió conocer la real ingesta del suplemento por parte de la embarazada; lo datos de la historia clínica prenatal constituyen una aproximación indirecta al seguimiento de la prescripción médica.

Se halló una correlación positiva y significativa entre la $\mathrm{Hb}$ de primer trimestre con el IMC pregestacional lo que se demuestra la importancia del buen estado nutricional desde antes de la concepción, que asegure adecuados niveles de $\mathrm{Hb}$ y una respuesta fisiológica a las altas demandas de este período $(12,15,17)$. De otro lado se encontró una correlación positiva y significativa entre la concentración de $\mathrm{Hb}$ inicial y la del tercer trimestre, esto permite demostrar la influencia de un buen nivel inicial de esta proteína, sobre un menor impacto de los altos requerimientos materno-fetales en la concentración de $\mathrm{Hb}$ al final de la gestación $(27,28)$. Se puede inferir la importancia de la suplementación con $\mathrm{Fe}$, para garantizar que en dicho período se controle su deficiencia y se mantenga una producción adecuada de $\mathrm{Hb}$, para asegurar el crecimiento y desarrollo fetal y prevenir la anemia $(27,29,30)$.

Entre las limitantes de este estudio debe tenerse en cuenta que el cálculo del tamaño de muestra, se basó en un estudio marco, donde la variable de interés fue la $\mathrm{Hb}$ como variable independiente que afecta el peso al nacer y del cual, se derivaron posteriormente, los objetivos de este, donde la $\mathrm{Hb}$ se asumió como variable dependiente de los factores sociodemograficos y gestacionales de las gestantes. Una recomendación para futuros trabajos, consiste en calcular el tamaño de muestra para un estudio de correlación. Además, para profundizar mejor en el análisis de resultados, se debe hacer un estudio comparativo por entidades prestadoras de salud públicas y privadas, que permitan una mayor diferenciación de las condiciones sociodemográficas de las embarazadas.

Otra limitante para la comparabilidad de los resultados de esta investigación radica en que la mayoría de los trabajos realizados en torno al comportamiento de la $\mathrm{Hb}$ gestacional, se han enfocado a la detección de aspectos que condicionan la aparición de anemia, en cambio, son pocos como éste, donde se pretendió estudiar la concentración de $\mathrm{Hb}$ gestacional de acuerdo a variables sociodemográficas y gestacionales de la población.

En conclusión, este estudio destaca que el déficit nutricional previo al embarazo es un factor asociado a 
la baja concentración de hemoglobina durante la gestación; este hecho hace prioritarias las acciones que en materia de salud pública buscan fortalecer la vigilancia nutricional de la mujer en el ciclo reproductivo, con énfasis durante la gestación, por medio de un adecuado programa de control prenatal, donde se fortalezca el seguimiento y el cumplimiento de la norma 412 (31), que busca detectar oportunamente, las alteraciones del embarazo. En este sentido, se deben fortalecer la valoración y el seguimiento nutricional a la embarazada y propender por la adherencia a hábitos de autocuidado y a la ingesta del suplemento de Fe y acido fólico para asegurar el bienestar nutricional del binomio madre-hijo.

\section{RESUMEN}

Objetivo: Explorar la asociación de factores sociodemográficos y gestacionales con la concentración de hemoglobina. Metodología: Estudio descriptivo transversal en 336 embarazadas de la Red Hospitalaria Pública de Medellín, 2007; se obtuvieron datos socio-demográficos, gestacionales y hemoglobina. Para el análisis se utilizó T de Student, ANOVA y correlación de Pearson. Resultados: el promedio de edad fue $24 \pm 6$ años, 67,6\% había cursado secundaria, $62,2 \%$ tenía un compañero estable, la asistencia a los controles prenatales fue $6 \pm 1$, peso pregestacional: $54,4 \pm 8,2 \mathrm{~kg}$, hemoglobina: $13,0 \pm 1,0 \mathrm{~g} / \mathrm{dL}$ en el primer trimestre, $11,9 \pm 1,1 \mathrm{~g} / \mathrm{dL}$ en segundo y $12,2 \pm 1,1$ $\mathrm{g} / \mathrm{dL}$ en tercero. Se encontró una diferencia significativa entre la hemoglobina por IMC pregestacional $(\mathrm{p}=0,035)$. La hemoglobina del primer trimestre correlacionó con el IMC pregestacional $(\mathrm{r}=0,178$ y $\mathrm{p}=0,028)$ y con la hemoglobina del tercer trimestre $(\mathrm{r}=0,356 \mathrm{y} \mathrm{p}=0,000)$. Conclusión: El IMC pregestacional, se asoció con la concentración de hemoglobina del primer trimestre y correlacionó con la hemoglobina final de la gestación.

Palabras clave: Gestación, hemoglobina, anemia, condiciones sociales, atención prenatal.

Dirigir la correspondencia a:

Profesora

Luz Stella Escudero Vásquez

Escuela de Nutrición y Dietética

Universidad de Antioquia, Colombia

Carrera 75\# 65-87, Bloque 44, Oficina 113

E-mail: lescud@pijaos.udea.edu.co

Agradecimientos: El grupo de investigación agradece la colaboración prestada por la Red Hospitalaria Pública de Medellín, sus funcionarios, la Escuela de Nutrición y Dietética y Laboratorios Laproff S.A. al brindar el apoyo logístico y financiero que permitieron la realización de este proyecto, igualmente al profesor
Alejandro Estrada por su acompañamiento en el análisis estadístico.

Conflicto de intereses: Los autores declaran que no existió conflicto de intereses entre el investigador, la institución, el laboratorio y las gestantes, por tratarse de información de fuente secundaria; además se aclara que el apoyo de Laboratorios Laproff, no influyó de manera alguna en el análisis de los datos, ni en su publicación.

\section{BIBLIOGRAFÍA}

1. Milman N. Prepartum anaemia: prevention and treatment. Ann Hematol, 2008; 87: 949-59.

2. Villares Álvarez I, Fernández Águila JD, Avilés Martínez M, Mediaceja Vicente O, Guerra Alfonso T. Anemia y deficiencia de hierro en embarazadas de un área urbana del municipio Cienfuegos. Rev Cuba Obstet Ginecol, 2006; 32: 1-6.

3. Boccio J, Salgueiro J, Lysionek A, Zubillaga M, Goldman C, Weill R, et al. [Iron metabolism: current concepts of an essential micronutrient]. Arch Latinoam Nutr, 2003; 53: 119-32.

4. Parra Sosa BE. Evaluación bioquímica del estado nutricional del hierro y el folato en un grupo de mujeres gestantes participantes del programa MANA para la vida. En: Restrepo S, ed. Alimentación y Nutrición de la Mujer Gestante: Diagnóstico y lineamientos para la acción. Medellín: Divegráficas Ltda; 2007. p. 217-43.

5. Mardones F, Duran E, Villarroel L, Gattini D, Ahumada D, Oyarzun F, et al. Maternal anemia in Concepcion province, Chile: association with maternal nutritional status and fetal growth. Arch Latinoam Nutr 2008; 58: 132-8.

6. Piña Baca F, La Torre Chivilchez L, Aylas Limache W. Comportamiento, actitudes y prácticas de alimentación y nutrición en gestantes. Rev Per Obst Enf 2007; 3: 17-21.

7. Yip R. Significance of an abnormally low or high hemoglobin concentration during pregnancy: special consideration of iron nutrition. Am J Clin Nutr 2000; 72: 272S-9S.

8. Tracer DP. Reproductive and socio-economic correlates of maternal haemoglobin levels in a rural area of Papua New Guinea. Trop Med Int Health 1997; 2: 513-8.

9. USAID, The World Bank, UNICEF, PAHO, FAO. Taking action: developing a strategy for anemia prevention and control. Lombardi K. and Wharton C. (eds). In: Anemia Prevention and Control: What works? Part I. Washington: PHNI and MEDS ; 2003: p 25-44. 
10. Uauy R, Athalah E, Barrera C, Behenke E. Alimentación y nutrición durante el embarazo. En: Uauy R, Athalah E, Barrera C, Behenke E, eds. Guías de alimentación para la mujer. Chile: Universidad de Chile. 2001: p. 53-74.

11. Olivares M, Walter T. Causas y consecuencias de la deficiencia de hierro. Rev Nutr [internet] 2004 mar [Citado en sep 6 de 2009]; 17: 5-14. Disponible en: http://www.scielo.br/scielo.php?pid $=$ S1415-52732004000100001\& script $=$ sci $\_$arttext \&lng=es:Doi:10.1590/S1415-52732004000100001

12. Becerra C, Gonzales GF, Villena A, de la Cruz D, Florian A. Prevalence of anemia in pregnancy, $\mathrm{Pu}$ callpa Regional Hospital, Perú. Rev Panam Salud Pública, 1998; 3: 285-92.

13. UNICEF, ONU, OMS. Iron Deficiency Anaemia: Assessment, Prevention and Control. A guide for programme managers. Washington: OMS; 2001: 99-101.

14. Zimmermann MB, Hurrell RF. Nutritional iron deficiency. Serie Lancet, 2007; 370: 511-20.

15. Gyorkos T, Shenker H, Larocque R, Ugaz M, Gotuzzo E. Sociodemographic and dietary correlates of anemia in pregnant women in Perú. Eco Food Nutr, 2004; 43: 497-516

16. Rodríguez Ganen O, Fernández Monagás SA, Fernández Manzano E, Rodríguez Acosta T, Gazapo Pernas R, Cutié León E, et al. Factores que inciden en la anemia ferropénica de la embarazada. Rev Cuba Farm, 2002; 36: 176-81.

17. Barba-Oropeza F, Cabanillas-Gurrola J. Factores asociados a la anemia durante el embarazo en un grupo de gestantes mexicanas. Arch Med Fam, 2007; 9: 170-5.

18. Van Den Broek NR, Rogerson SJ, Mhango CG, Kambala B, White SA, Molyneux M. Anaemia in pregnancy in southern Malawi: prevalence and risk factors. Br J Obstet Gynaecol. 2000; 107: 445-51.

19. Carballo JA, Garayalde León JA. Comportamiento de la anemia en el embarazo en un área de salud. [Monografía en Internet] 2009 [Citada en jun 20 de 2009]. Disponible en: http://www.monografias.com/ trabajos70/comportamiento-anemia-embarazoarea-salud/comportamiento-anemia-embarazoarea-salud2.shtml

20. Bharati P, Som S, Chakrabarty S, Bharati S, Pal M. Prevalence of anemia and its determinants among nonpregnant and pregnant women in India. Asia
Pac J Public Health. 2008; 20: 347-59.

21. Bodnar LM, Siega-Riz AM, Arab L, Chantala K, McDonald T. Predictors of pregnancy and postpartum haemoglobin concentrations in low-income women. Public Health Nutr 2004 Sep; 7: 701-11.

22. Sotero Salgueiro GA, Sosa Fuertes CG, Domínguez Rama Á, Alonso Telechea J, Medina Milanesi R. El estado civil materno y su asociación con los resultados perinatales en una población hospitalaria. Rev Med Urug [Internet] 2006 mar [Citado en sep 8 de 2009]; 22: 59-65. Disponible en: http://www.scielo. edu.uy/scielo.php?script=sci_arttext\&pid=S0303$32952006000100009 \& \operatorname{lng}=\mathrm{es} \& \mathrm{nrm}=\mathrm{iso}>$. ISSN 0303-3295.

23. Parra BE, Manjarres LM, Gomez AL, Alzate DM, Jaramillo MC. Evaluación de la educación nutricional y un suplemento para prevenir la anemia durante la gestación.]. Biomedica 2005 Jun; 25: 211-9.

24. Tarín A LC, Gómez A D, Jaime P JC. Anemia en el embarazo. Estudio de 300 mujeres con embarazo a término. Medicina Universitaria. 2003; 5: 149-53.

25. Da Silva Ferreira H, Moura FA, Cabral J CR. Prevalência e fatores associados a anemia em gestantes da região semi-árida do Estado de Alagoas. Rev Bras Ginecol Obstetr 2008; 30: 445-51.

26. Uzcátegui $\mathrm{O}$, Toro Merlo J. Metas del milenio y salud materna. Rev Obstet Ginecol Venez 2009; 69: 1-3.

27. Paiva A de A, Rondó PHC, Pagliusi RA, Latorre M do RDO, Cardoso MAA, Gondim SSR. Relationship between the iron status of pregnant women and their newborns. Rev Saúde Pública 2007; 41: 321-7.

28. Allen L H. Anemia and iron deficiency: effects on pregnancy outcome. Am J Clin Nutr 2000 May; 71: 1280S-4S.

29. Bhutta ZA, Ahmed T, Black RE, Cousens S, Dewey $\mathrm{K}$, Giugliani E., et al. What works? Interventions for maternal and child undernutrition and survival En: Maternal and Child Undernutrition. Serie Lancet 2008; 371: 417-40.

30. Vera GL, Quintal DR, González MP, Vázquez CG. Prevalencia de anemia ferropénica en mujeres embarazadas rurales en Valladolid, Yucatán, México. Ginecol Obstet Mex 2009; 77: 544-9.

31. Ministerio de Salud. Resolución 00412 de febrero 25 de 2000. Bogotá [Internet] [Citada en 25 de julio de 2011]. Disponible en: http://www.nacer.udea.edu. co/pdf/libros/guiamps/guias03.pdf. 